\title{
Role of Conferences in Shaping the Field of HCI
}

\author{
Jan Gulliksen ${ }^{1}$ (panel coordinator), Simone Diniz Junqueira Barbosa ${ }^{2}$, \\ Anirudha Joshi ${ }^{3}$, Shaun Lawson ${ }^{4}$, Philippe Palanque ${ }^{5}$ \\ ${ }^{1}$ KTH Royal Institute of Technology, Stockholm, Sweden \\ gulliksenekth.se \\ ${ }^{2}$ PUC Rio, Brazil \\ simonedinf.puc-rio.br \\ ${ }^{3}$ IIT Bombay, Mumbai, India \\ anirudha@iitb.ac.in \\ ${ }^{4}$ LiSC, University of Lincoln, U.K. \\ slawsonelincoln.ac.uk \\ ${ }^{5}$ ICS-IRIT, University of Toulouse, France \\ palanquedirit.fr
}

\begin{abstract}
The panel will discuss the role various conferences have played in developing the field of HCI in academic research and industrial practice. It is composed of people who have experience in organising HCI conferences in different parts of the world. It provides a platform to the participants to think and reflect about what they are doing when attending a conference, what their expectations are and how it impacts positively their knowledge, work and career.
\end{abstract}

Keywords. Human-computer interaction, conferences

\section{Introduction}

Why go to an international conference? Is it because you see it as a good way to justify that your university pays you to travel the world? Is it because you like to hang out and drink beers with a lot of fun people? Is it because you love to be on the stage and talk about yourself and how good you are? Is it because you think you will get new contacts and sneak you way into a research position that you otherwise would not be able to get? Or do you think it is a way to get famous?

This panel aims at scrutinizing whether it is sustainable to continue polluting the planet by making big crowds travel all across the world for conferences. It aims at discussing whether the acceptance of papers in the field is based on excellence, or whether the system is simply corrupt. It aims at discussing whether it is a business for conference organizers to make money on the side or for big international organizations to colonize the world with their views and perspectives.

In the last two decades, HCI conferences have generally done well. Many conferences have grown in numbers. Some have increased their frequency. Some are broad, others are focussed. Some have explored new geographies, new cultures. New, smaller con- 
ferences have emerged, either specialising in a domain or catering to the interests of a local community. Meanwhile, the world has gotten more connected by digital technologies, and more people are now online than ever were. Among other changes, virtual meetings have become more of a norm than an exception.

This is an interesting juncture to seek out what lies ahead and also to ponder about what we might be missing out. Many new questions are being raised that are interesting and relevant. Must all conferences have papers? What is an optimal size of a conference $-50,500,5,000$, or 50,000? Are we focussing too much on the social programme? On the other hand, would it still be a "conference" if everyone were to participate remotely? Do we need to worry about the relatively infrequent participants industry professionals, students, those with disabilities, new parents (especially mothers), and those from developing countries?

Worldwide, researchers, research units, and Universities are increasingly being subject to research evaluation exercises which often lead to far-reaching implications around funding, promotion and recruitment. Where do conferences fit within such evaluation programs? How are even the best conference publications viewed in such processes? How is the field of $\mathrm{HCI}$ influenced by other disciplines with different publication practices?

This panel consists of people with experience in organising small and large conferences in different parts of the world. They will introspect about the historical role of conferences in shaping the field of HCI and ponder about how they see conferences evolving over the next few years.

\section{Panel members}

Jan Gulliksen is professor in Human Computer Interaction and the Dean of the School of Computer Science and Communication at KTH Royal Institute of Technology, in Stockholm, Sweden. He is in charge of a school comprising 350 employed staff, educating about 1200 students each year. Jan teaches various courses in Human Computer Interaction, Usability and User-Centred Design at undergraduate and postgraduate level and does research on Usability, accessibility and usercentred design in practice. He has been associated with various conferences including CHI, INTERACT, NordiCHI, HCSE and others. He is the current chair of IFIP TC13 on HCI which is the organization in charge of the INTERACT conference series. He is also, as the founder of NordiCHI chairing the organization behind the NordiCHI conference.

Simone Diniz Junqueira Barbosa is associate professor at the Department of Informatics, PUC-Rio, Brazil. Level 2 researcher in CNPq (National Council for Scientific and Technological Development in Brazil) and chair of the Special Interest Group on Human-Computer Interaction of the Brazilian Computer Society, she has served as chair in several national and international conferences, e.g., Latin-American Conference on Human-Computer Interaction, CHI, INTERACT, EICS, and IHC.

Anirudha Joshi is professor in the interaction design stream in the Industrial Design Centre, IIT Bombay, India. His research is in the area of interaction design for people 
in developing countries. Much of his recent work is in designing text input mechanisms for Indian languages. He also works in the cusp between human-computer interaction and software engineering. He has been IFIP TC13 representative from India. $\mathrm{He}$ has been active in organising several conferences including India HCI, APCHI, INTERACT, and CHI.

Shaun Lawson is Professor of Social Computing at the University of Lincoln in the UK where he directs the Lincoln Social Computing (LiSC) Research Centre. His research interests are mainly centred upon the design, use and implications of interactive social applications and social media. In particular he is interested in how these platforms can be used for political, activist and persuasive aims. He has extensive experience of conference management and organisation in $\mathrm{HCI}$ and social computing settings; most recently he was General Chair of British HCI 2015 and served on the organising committees for CHI in both 2014 and 2015.

Philippe Palanque is professor in Computer Science at the University Toulouse 3 France. He works in the area of interactive systems engineering with a focus on the dependability, safety and usability of critical systems. He is secretary of the IFIP Working group 13.5 on Human Error Safety and System Development and chaired with Chris Johnson the track on that topic within the IFIP WCC 2004. He was the general co-chair of CHI 2014 and full paper co-chair of INTERACT 2015 and has been on the SIGCHI executive committee in charge of specialized conferences since 2007. 